\title{
La hidroquinina resultó segura y efectiva para los calambres musculares
}

\author{
Randomised controlled trial of hydroquinine in muscle \\ cramps. Jansen PHP, Veenhuizen KCW, Wesseling AIM et al. Lan- \\ cet 1997;349:528-32
}

\section{Objetivo}

Evaluar la seguridad y eficacia de la hidroquinina (HQ) para el tratamiento de adultos con calambres musculares frecuentes.

Diseño

Estudio randomizado, doble ciego, controlado, de grupos paralelos.

Lugar

Países Bajos

\section{Pacientes}

Se incluyeron 112 adultos sanos mayores de 18 años con tres o más calambres por semana en reposo o actividad. Criterios de exclusión: 1) Estar tomando medicación para calambres u otra droga que tenga interacción con HQ; 2) Calambres de más de 10 minutos; 3)Consumo de drogas o alcohol; 4) Contraindicaciones o alergia a HQ; 5) Objeción del médico de cabecera; 6)Embarazo o lactancia; 7) Mujeres en edad fértil sin método anticonceptivo. El estudio tuvo 3 fases consecutivas de dos semanas de duración: 1) Evaluación de frecuencia basal de calambres (calificación); 2) Tratamiento con HQ o placebo; 3) Evaluación de los efectos posteriores (lavado). Los resultados se analizaron en 102 pacientes (91\%).

\section{Intervención}

HQ $200 \mathrm{mg}$ con la cena y $100 \mathrm{mg}$ al acostarse $(\mathrm{n}=54)$ vs placebo $(n=58)$.

\section{Medición de resultados principales \\ Se evaluaron el número de calambres y la cantidad de días con calambres. Otros: duración, severidad, adherencia y efectos ad-} versos.

\section{Resultados Principales}

El análisis fue por intención de tratar*. Ambos grupos tenían características similares al inicio del estudio. En ambos grupos disminuyó el número de calambres y de días con calambres en la fase de tratamiento, pero esta mejoría fue mayor en el grupo HQ. Comparando la fase de calificación con la de tratamiento, el grupo HQ tuvo una mediana* de 8 (IC 95\% 7-12) calambres menos y una mediana de 3 (IC 95\% 1-4) días menos con calambres mientras que el grupo placebo reportó sólo 3 (IC 95\% 0-5) calambres menos y 1 (IC $95 \%$ 0-5) día menos con calambres. En el grupo HQ 32 pacientes $(65 \%)$ tuvieron una reducción del 50\% del número de calambres, y ésta disminución se observó en 10 pacientes (19\%) del grupo placebo. Se comprobó un efecto sostenido al suspender el tratamiento. No hubo diferencias en la adherencia al tratamiento, en la severidad y en la duración de calambres. La HQ tuvo más efectos adversos leves (boca seca, gusto amargo en 10 pacientes)

\section{Conclusiones}

La HQ $300 \mathrm{mg} /$ día fue segura y más efectiva que el placebo para prevenir calambres frecuentes en el corto plazo. El efecto terapéutico se observó más allá del periodo de tratamiento.

Fuente de financiamiento: ASTA medica (fabricante de hidroquinina)

\section{Comentario}

Los calambres musculares son un problema común en la práctica de atención primaria, sin embargo han tenido poca atención en la literatura médica. Muchos tratamientos fueron sugeridos incluyendo masajes, caminatas, elongación muscular, y medicaciones como sulfato de quinina, verapamilo y vitamina $E$ (1-4). En nuestro país es de practica corriente el uso de magnesio para tratar los calambres de miembros inferiores. De todas estas intervenciones, la única que mostró efectos beneficiosos fue la quinina y sus derivados, que en dos recientes estudios bien diseñados demostró una reducción significativa en el número y severidad de los calambres (4). A pesar de sus beneficios, el uso de esta medicación está limitado por sus efectos adversos como trombocitopenia y cinconismo (síndrome que incluye nauseas, vómitos, acúfenos, alteraciones visuales y disminución de la audición). En este estudio se utilizó hidroquinina (un derivado de la quinina) $300 \mathrm{mg}$ diarios versus placebo, encontrándose una reducción significativa en el número de calambres y en el número de días con calambres. Hubo mas efectos adversos leves en el grupo HQ, en cuanto a los efectos adversos severos que son poco frecuentes, no pudieron ser evaluados por falta de poder (poco número de pacientes). Con respecto a la tan difundida práctica en nuestro medio de tratar con magnesio este problema, se realizó en el Hospital Italiano de Buenos Aires, un estudio controlado, randomizado, doble ciego en el cual no se observaron diferencias con respecto al placebo para aliviar los calambres nocturnos (5). Ambos grupos mostraron similar mejoría lo que habla de un importante efecto placebo. Teniendo en cuenta que los calambres son muchas veces autolimitados y variables en el tiempo; frente a un paciente con calambres que no responde al tratamiento no farmacológico o tal vez a algún tratamiento placebo; deberíamos plantearnos la utilización de sulfato de quinina o hidroquinina considerando los riesgos marginales de este tra- tamiento. La dosis inicial es de $200 \mathrm{mg}$ administrados por la noche y en caso de falta de respuesta se puede aumentar a $300 \mathrm{mg}$.

*Ver glosario

Dr. Ricardo Frusso Unidad de Medicina Familiar y Preventiva

Hospital Italiano de Buenos Aires

Referencias :

1. Goroll A, May LA, Mulley A. Primary Care Medicine. Third edition. JB Lippincott Company. $1995: 754-6$

2. Baltodano N Gallo BV, Weidler DJ. Verapamil vs quinine in recumbent nocturnal leg cramps in the elderly. Arch intern Med. 1988 : $148: 1969$. 70

3. Mc Gee SR. Muscle cramps. Arch Intern Med. 1990;150:511-518.

4. Priscilla SC, Eric AS. Treatment of nocturnal leg cramps. Arch Intern Med.1992;152:1877-80 5. Frusso R, Zárate M, Augustovski F y cols (abstract). Magnesium for nocturnal leg crampsa randomized crossover trial.. En prensa Journal of General Internal Medicine, 1998. 